\title{
Synthesis and Characterization of Superhydrophobic Films with Raspberry-Like Silica Nanoparticles as Functional Coatings ${ }^{+}$
}

\author{
Maria Antonia Tănase 1,*, Adina Raducan 1, Petruța Oancea ${ }^{1}$, Cristina Scomoroscenco ${ }^{2}$, \\ Elvira Alexandrescu ${ }^{2}$, Bogdan Trică ${ }^{2}$, Cristina Lavinia Nistor ${ }^{2}$, Cristian Petcu ${ }^{2}$, Alina Popescu ${ }^{3}$, \\ Ioana Rodica Stănculescu ${ }^{4}$ and Ludmila Otilia Cinteza ${ }^{1}$ \\ 1 University of Bucharest, Faculty of Chemistry, 4-12 Regina Elisabeta Blvd., 030118 Bucharest, Romania; \\ adina.raducan@g.unibuc.ro (A.R.); petrutaoancea73@yahoo.com (P.O.); \\ ocinteza@gw-chimie.math.unibuc.ro (L.O.C.) \\ 2 National Research and Development Institute for Chemistry and Petrochemistry-ICECHIM, \\ 060021 Bucharest, Romania; scomoroscencocristina@gmail.com (C.S.); \\ elviraalexandrescu@yahoo.com (E.A.); trica.bogdan@gmail.com (B.T.); \\ lc_nistor@yahoo.com (C.L.N.); cpetcu@icf.ro (C.P.) \\ 3 National Research \& Development Institute for Textiles and Leather, 030508 Bucharest, Romania; \\ alinapopescuro@yahoo.com \\ 4 Horia Hulubei-NIPNE, P.O. BOX MG-6 Ilfov, Romania; ioana.stanculescu@ninpne.ro \\ * Correspondence: maria.a.tanase@gmail.com \\ + Presented at the 16th International Symposium "Priorities of Chemistry for a Sustainable Development" \\ PRIOCHEM, Bucharest, Romania, 28-30 October 2020.
}

Published: 13 November 2020

Keywords: functional coatings; superhydrophobic; raspberry-like silica nanoparticles

Superhydrophobic materials of innovative products were intensively studied during the last few decades, and many methods for their fabrication have been developed [1]. The basic concept to obtain ultra and superhydrophobic properties is the simultaneous presence of a low energy material and suitable roughness on the surface. Biomimetic strategy to achieve superhydrophobic properties that mimic lotus leaf consists in special hierarchical morphology replication. The paper presents a facile synthesis of filmogenic material, based on silica nanoparticles with unusual morphology (raspberrylike) and the changes in wettability of various solid substrates treated with the proposed coating.

Functional nanomaterials have been prepared by embedding silica nanoparticles with raspberry-like morphology in polysiloxane matix. Silica nanoparticles have been synthesized using a simple sol-gel method, at room temperature, using methyl triethoxysilane (TMES) as silane precursor. Raspberry-like silica particles have been obtained by the self-assembling process of oppositely charged $\mathrm{SiO}_{2}$ nanoparticles, functionalized with various organo-modified silane reagents. $\mathrm{SiO}_{2}$ nanoparticles were characterized from the point of view of size, surface potential, and shape using dynamic light scattering (DLS), scanning electron microscopy (SEM), and transmission electron microscopy (TEM). Filmogenic nanomaterial was investigated in terms of composition using FTIR, morphology using SEM and wettability by using contact angle measurements, respectively.

The coatings deposited on model paper and textile exhibits a static contact angle for water of 1550 and 1580 with contact angle hysteresis less than 80 .

Double hierarchical morphology of nanomaterial deposited was evidenced by SEM images that confirm the Atomic Force Microscopy (AFM) results of high roughness of coated films.

The presence of silica nanoparticles with raspberry-like morphology in the coating material leads to a structure of the final film that closely resembles to the hierarchical aspect of natural 
superhydrophobic surfaces. A simple, cost effective way to produce functional nanomaterials for superhydrophobic modification of surfaces was obtained.

Acknowledgments: This work was supported by grans of the Romanian National Authority for Scientific Research and Innovation, CCCDI-UEFISCDI, project number PN-III-P1-1.2-PCCDI-2017-0743(PC5) and project number PN-III-P1-1.2-PCCDI-2017-0428 (PC2), within PNCDI III.

\section{Reference}

1. Nishimoto, S.; Bhushan, B. Bioinspired Self-Cleaning Surfaces with Superhydrophobicity, Superoleophobicity, and Superhydrophilicity. RSC Adv. 2013, 3, 671-690.

Publisher's Note: MDPI stays neutral with regard to jurisdictional claims in published maps and institutional affiliations.

(c) 2020 by the authors. Licensee MDPI, Basel, Switzerland. This article is an open access article distributed under the terms and conditions of the Creative Commons Attribution (CC BY) license (http://creativecommons.org/licenses/by/4.0/). 